\title{
Proton versus photon deep inspiration breath hold technique in patients with hodgkin lymphoma and mediastinal radiation
}

\section{A Planning Comparison Of Deep Inspiration Breath Hold Intensity Modulation Radiotherapy And Intensity Modulated Proton Therapy}

Christian Baues $^{1 *}$, Simone Marnitz ${ }^{1 \dagger}$, Andreas Engert ${ }^{2,3}$, Wolfgang Baus ${ }^{1}$, Karolina Jablonska ${ }^{1}$, Antonella Fogliata ${ }^{4}$, Andrés Vásquez-Torres ${ }^{1}$, Marta Scorsetti ${ }^{4,5}$ and Luca Cozzi ${ }^{4,5}$

\begin{abstract}
Background: The benefits of proton therapy in the treatment of patients with Hodgkin lymphoma $(\mathrm{HL})$ are controversially discussed. Therefore we compared intensitiy modulated proton therapy (IMPT) with intensity modulated radiotherapy (IMRT), in the form of volumetric modulated arc therapy (VMAT) in patients with Hodgkin lymphoma $(\mathrm{HL})$, through a comparative treatment planning study.

Methods: Radiation plans for 21 patients with Hodgkin Lymphoma (HL) were computed for IMPT and deep inspiration breath hold (DIBH) VMAT. Plans were optimized and computed assuming deep inspiration breath holding conditions. Dosimetric comparison on standard metrics from dose volume histograms was performed to appraise the relative merits of the two techniques, while proton plan robustness was assessed by re-computing the dose distribution of each plan by varying the Hounsfield Units to stopping power calibration by applying $a \pm 3$ and $4 \%$ error.
\end{abstract}

Results: DIBH-VMAT and IMPT both provided excellent coverage, conformity and heterogeneity of the clinical target volume (CTV) and planning target volume (PTV). IMPT reduced mean doses to the breasts, lungs, heart and normal tissue by 38-83\%. IMPT significantly reduced mean doses to the heart to $<5$ Gy despite bulky mediastinal disease and decreased breast doses in female patients to $<1 \mathrm{~Gy}$. Despite the simulated 3 and $4 \%$ miscalibration errors, no remarkable or measurable impact was observed on the organs at risk (OARs).

Conclusions: This is the first comparison between DIBH-VMAT and IMPT in HL treatment. We could demonstrate statistically significant decreases in all dose/volume metrics of the OARs. Regardless of the planning paradigm used, range uncertainties can substantially under dose the PTV, while perhaps not leading to clinically significant deterioration of CTV coverage. With the geometry applied no impact was observed for OARs, suggesting IMPT as a superior technique for potentially reducing future health risks for HL patients.

Keywords: Hodgkin lymphoma, Proton beam therapy, Range uncertainties, VMAT

\footnotetext{
*Correspondence: Christian.Baues@uk-koeln.de

${ }^{+}$Christian Baues and Simone Marnitz contributed equally to this work.

${ }^{1}$ Medical Faculty, Department of Radiation Oncology, Cyberknife Center and

Radiation Reference Center of the GHSG, University of Cologne, Kerpener Str.

52, 50937 Köln, Cologne, Germany

Full list of author information is available at the end of the article
}

(c) The Author(s). 2018 Open Access This article is distributed under the terms of the Creative Commons Attribution 4.0 International License (http://creativecommons.org/licenses/by/4.0/), which permits unrestricted use, distribution, and reproduction in any medium, provided you give appropriate credit to the original author(s) and the source, provide a link to the Creative Commons license, and indicate if changes were made. The Creative Commons Public Domain Dedication waiver (http://creativecommons.org/publicdomain/zero/1.0/) applies to the data made available in this article, unless otherwise stated. 


\section{Background}

Multi-agent chemotherapy followed by radiation is the standard treatment for patients with Hodgkin Lymphoma (HL). However, even after mediastinal irradiation, follow-up has demonstrated considerable long-term morbidity and mortality caused by cardiovascular disease, or lung and breast cancer [1-7]. Two large randomized studies, the H10 trial of EORTC and the RAPID trial, attempted to eliminate radiotherapy in favorable stage $\mathrm{HL}$, but both failed to meet the predefined inferiority margin when radiotherapy was omitted $[8,9]$. Options to decrease toxicity include reducing the radiation dose, the intensity of chemotherapy, the radiation target volume, and inadvertent radiation doses to healthy tissue [10-14]. For HL treatment the chosen target volume has progressively been reduced from total nodal to extended field, then involved field and finally involved node concepts [8, 15]. Furthermore, the International Lymphoma Radiation Oncology Group (ILROG) contributed to standardizing the target volume definition and recommendations for treatment of HL patients, defining the involved site concept as a variation of involved node radiotherapy $[11,12,16]$. Changing the target volume concepts from involved field radiation (IFRT) to involved node (INRT), Weber et al. [17] showed significantly decreased excess relative risk for breast, lung and thyroid regardless of the radiation technique used. Nevertheless, the optimal technique, beam quality and delivery of radiation treatment are the subject of numerous comparative dose-planning studies and are still a matter of debate.

For treatment of mediastinal involvement in HL patients, proton, photon radiation with 3-D conventional techniques versus intensity modulated radiation (IMRT) or volumetric arc techniques (VMAT) have been compared [16-23]. Concerning protons, Hoppe et al. [24] reported about 15 patients who received involved node proton therapy. With a median follow-up of 37 months only two negative events occurred (one relapse and one histology transformation) while the 3-year relapse free survival was $93 \%$ and the event free survival was $87 \%$. No severe acute or late toxicity was observed. In a more recent study [25] Hoppe reports the outcome of the treatment of 138 patients. The 3-year relapse free survival rate was $92 \%$ for all patients and $96 \%$ for the adults (87\% for pediatric patients).

Whereas optimized VMAT reduced heart disease, it increased the lung dose and the probability of inducing cancer. Furthermore, results from comparative studies are influenced by dose constraint definitions, different planning goals for various organs at risk (OARs), and heterogeneous anatomical [20], supporting an individualized approach for each patient involving routine planning comparisons [14]. Special beam configurations and patient positioning lead to a significant dose reduction in the volume covered by 20 Gy and 30 Gy (V20, V30) to all critical structures: to the breasts, total lung, heart, left and right ventricle, coronary vessels and to the spinal cord $[10,20]$. Moreover, radiation in deep inspiration breath hold (DIBH) reduces the excess risks of myocardial infarction and lung cancer compared with free breathing [23]. Valid clinical data about the reduction of the tumor induction are still missing.

Other publications focused on the comparison of photon techniques versus $3 \mathrm{D}$ proton treatment (3D-PRT) $[26,27]$. However, no data are available from randomized trials comparing the oncologic outcome for proton radiotherapy (PRT). Data of long-term toxicity are also lacking [4]. The majority of publications only evaluated planning comparisons. Protons deposit most of their radiation dose in tissue near the target volume, depending on the energy. A reduced entrance dose may contribute to a lower dose to healthy tissue. Thus PRT allows low dose sparing with better protection of breast tissue comparing to IMRT/VMAT, which is an important factor to consider when treating Hodgkin lymphomas in female patients [26, 28]. One phase II study [18] on PRT versus $3 \mathrm{D}$ proton and IMRT photon radiation for INRT in Stage I-IIIA HL patients demonstrated the lowest mean dose to the heart, lungs, and breasts with PRT, and considerable mean dose reduction for PRT compared to photon IMRT for the heart, lungs and breasts and healthy tissue $[19,21]$.

The question concerning which parameters (sub)-volumes versus mean dose; high dose volume versus low dose volume is of higher clinical importance is still unanswered and depends on many factors. Compared with passive-beam PRT and conformal 3DRT, helical tomotherapy (HT) achieved better protection of the lungs for doses above 15 Gy. However, mean doses to breasts, lung tissue and heart with PRT were significantly lower compared to 3DRT and HT [28].

A study with 22 patients and a case series have also recently addressed this question. Both came to the conclusion that a further reduction of the burden on risk organs can be achieved through the use of IMPT. Furthermore, the expected reduction in life years can be assumed according to a mathematical model [29, 30]. Our aim was to perform a dose comparative study, using intensity modulated proton (IMPT) and volumetric modulated arc therapy (VMAT) with photons with regard to target volumes and OARs. Additionally, the perturbation of proton dose (PDP), secondary to isocenter calibration errors, was estimated in this planning comparison.

\section{Methods}

\section{Target volume and OAR delineation}

In this single center analysis, all patients with Hodgkin lymphoma and mediastinal involvement were consecutively included in the period from July 2014 to 
March 2016 and irradiated with a Photon VMAT plan. As part of the study, an IMPT plan was then calculated for each patient and a comparative evaluation was made. Twenty-one patients with a mean age of 34 years (16 women and 5 men) with certain mediastinal involvement of HL in stages I-IV treated at Cologne University Hospital were included in this study (Table 1). The target volumes were delineated according to the guidelines of HL 16/17/18 studies of the German Hodgkin Study Group (GHSG). Regarding the stages delineations were performed following the IFRT or INRT definition in HD 16 and 17. Patients in the HD 18 study received local radiation in PET-positive areas of more than $2.5 \mathrm{~cm}$ after chemotherapy. The results in new structures that must be taken into account when setting and subscribing to the target volume. More specifically, the gross tumor volume (GTV) was the lymph node remnant (s) observed on the post-chemotherapy CT. The clinical tumor volume (CTV) was defined as the morphological volume of the initial mediastinal involvement with respect to treatment response and replacement of OAR's after chemotherapy. CTV included the GTV in patients with partial response after chemotherapy. Adding an $8-10 \mathrm{~mm}$ margin in the cranio-caudal axis and $6-8 \mathrm{~mm}$ in the lateral axis to the CTV derived the planning tumor volume PTV. The same experienced radiation oncologist delineated the OAR's. The same target volumes (CTV and PTV) were used for the optimization of the proton and photon plans. All CT scans were acquired in deep inspiration breath hold mode and all the plans were optimized on these datasets.

\section{Proton beam planning}

Intensity modulated proton plans were optimized to deliver 30 GyE to the PTV.

IMPT plans were created by an experienced physicist (L.C.) using beam spot scanning and intensity modulation realized by means of an inverse optimization process via tuning the spot energy and beam weights simultaneously. The ProBeam proton system (Varian Medical systems, Palo Alto, USA) was used as a source of beam data. The basic workflow for IMPT planning was as follows: calculating the beam line settings, optimizing the spot weights for all field simultaneously, post-processing the spot list (where a deliverable spot scanning sequence was generated), and calculating the final dose via summing all the scanning layers, accounting for differences in tissue properties within the patient. The beam arrangement chosen included three or four

Table 1 Involved lymph node areas of all patients

\begin{tabular}{|c|c|c|c|c|c|c|c|c|}
\hline & Cervical left & Cervical right & Supra/infraclav. Left & Supra/inrfaclav. Right & Upper mediastinum $^{\mathrm{a}}$ & Lower mediastinum $^{\mathrm{a}}$ & Right axilla & Left axilla \\
\hline Pat. 1 & - & - & - & - & $x$ & $x$ & - & - \\
\hline Pat. 2 & - & - & - & - & $x$ & $x$ & - & - \\
\hline Pat. 3 & - & - & - & - & $x$ & $x$ & - & - \\
\hline Pat. 4 & - & - & - & - & $x$ & $x$ & - & - \\
\hline Pat. 5 & $x$ & $x$ & $x$ & $x$ & $x$ & - & - & - \\
\hline Pat. 6 & - & - & - & $x$ & $x$ & & - & - \\
\hline Pat. 7 & $x$ & $x$ & $x$ & $x$ & $x$ & - & - & - \\
\hline Pat. 8 & - & $x$ & - & $x$ & $x$ & $x$ & - & - \\
\hline Pat. 9 & - & $x$ & $x$ & $x$ & $x$ & $x$ & - & $x$ \\
\hline Pat. 10 & - & - & - & - & $x$ & $x$ & - & - \\
\hline Pat. 11 & - & - & - & - & $x$ & $x$ & - & - \\
\hline Pat. 12 & - & - & $x$ & $x$ & $x$ & - & - & - \\
\hline Pat. 13 & - & - & - & - & $x$ & $x$ & - & - \\
\hline Pat. 14 & $x$ & X & $x$ & X & $x$ & $x$ & - & - \\
\hline Pat. 15 & - & - & X & $x$ & $x$ & - & - & - \\
\hline Pat. 16 & $x$ & X & X & X & $x$ & $x$ & $x$ & $x$ \\
\hline Pat. 17 & - & - & - & - & $x$ & $x$ & - & - \\
\hline Pat. 18 & - & X & $x$ & X & $x$ & $x$ & - & - \\
\hline Pat. 19 & - & X & - & $x$ & $x$ & $x$ & - & - \\
\hline Pat. 20 & - & - & - & - & $x$ & $x$ & - & - \\
\hline Pat. 21 & $x$ & X & $x$ & $x$ & $x$ & - & - & - \\
\hline
\end{tabular}

${ }^{a}$ The border between the upper and the lower mediastinum was defined by the tracheal bifurcation 
fields. For all patients 3 fields were defined as one posterior and two oblique-anterior. For some patients, a fourth anterior field was added to improve target coverage. The specific gantry angles were chosen on a patient per patient basis to minimize the volume of lungs crossed proximally to the target as well as for the other organs at risk. The Bragg peak distribution in depth was achieved using various pencil beam energies (with a nominal range from 50 to $170 \mathrm{MeV}$ ) and the weights of individual beams were optimized simultaneously for irradiation fields. The dose calculation for all proton plans was performed on a $2.5 \times 2.5 \times 2.5 \mathrm{~mm}^{3}$ grid. The Eclipse treatment planning system (Varian Medical systems, Palo Alto, USA) v.13.6 was used for this part of the study.

\section{Volumetric modulated arc therapy planning}

VMAT plans were optimized by A. V. and W. B. using the same constraints as for the IMPT planning. The planning CT was acquired with a Toshiba LG 16 row scanner with the patient holding their breath in deep inspiration. The deep inspiration breath hold status during $\mathrm{CT}$ acquisition was measured and documented using the Real-time Position Management (RPM v. 1.7, Varian Medical Systems (VMS), Palo Alto, USA).

\section{Dose comparison study}

Dose-volume histograms (DVHs) were calculated for the PTV and organs at risk (OARs): lungs, heart, breasts (for females patients) and spinal cord and healthy tissue defined as the fraction of body included in the CT scan minus the target volumes. For the heart the myocardium was also considered and defined as "heart wall". We defined it as a new parameter, which might be a better prognostic factor of long-term heart toxicity than commonly used heart mean dose. Dose constraints are shown in Table 2 for the target volumes and in Table 3 for the OARs.

Table 2 Summary of target volumes dosimetric analysis from Dose Volume histograms

\begin{tabular}{|c|c|c|c|c|}
\hline Parameter & Objective & IMPT & RA & $p$ (Wilcoxon) \\
\hline \multicolumn{5}{|c|}{ CTV: $481.3 \pm 358.5[108-1355] \mathrm{cm}^{3}$} \\
\hline Mean [Gy] & 30.0 & $30.1 \pm 0.2$ & $30.6 \pm 0.2$ & n.s. \\
\hline$D_{1 \%}[G y]$ & Minimize & $31.5 \pm 0.5$ & $32.0 \pm 0.5$ & 0.01 \\
\hline$D_{5 \%}-D_{95 \%}[G y]$ & Minimize & $2.0 \pm 0.3$ & $2.5 \pm 0.4$ & $<0.01$ \\
\hline$D_{98 \%}[\%]$ & $>28.5 \mathrm{~Gy}(95 \%)$ & $29.4 \pm 0.1$ & $28.8 \pm 0.4$ & 0.04 \\
\hline \multicolumn{5}{|c|}{ PTV: $881.8 \pm 597.0$ [215.7-2205.7] $\mathrm{cm}^{3}$} \\
\hline Mean [Gy] & 30.0 & 30.0 & 30.0 & - \\
\hline $\mathrm{D}_{1 \%}[\mathrm{~Gy}]$ & Minimize & $31.7 \pm 0.3$ & $32.0 \pm 0.4$ & 0.07 \\
\hline$D_{5 \%}-D_{95 \%}[G y]$ & Minimize & $2.3 \pm 0.3$ & $3.5 \pm 0.4$ & $<0.01$ \\
\hline$D_{95 \%}[\%]$ & >27Gy (90\%) & $28.8 \pm 0.3$ & $27.3 \pm 0.7$ & $<0.01$ \\
\hline
\end{tabular}

$D_{\mathrm{x} \%}$ dose received by the $\mathrm{x} \%$ of the volume, $V_{\mathrm{x} \%}$ volume receiving at least $\mathrm{x} \%$ of the prescribed dose, $\mathrm{Cl}$ ratio between the patient volume receiving at least $90 \%$ of the prescribed dose and the volume of the total PTV
Table 3 Summary of Organs at risk dosimetric analysis from Dose Volume histograms

\begin{tabular}{|c|c|c|c|c|}
\hline Parameter & Objective & IMPT & RA & $p$ (wilcoxon) \\
\hline \multicolumn{5}{|c|}{ Spinal cord: $41.6 \pm 15.6 \mathrm{~cm}^{3}$} \\
\hline$D_{1 \%}[G y]$ & Minimize & $15.9 \pm 5.622 \%$ & $20.3 \pm 5.2$ & $<0.01$ \\
\hline \multicolumn{5}{|c|}{ Left breast: $467.5 \pm 233.9 \mathrm{~cm}^{3}$} \\
\hline Mean [Gy] & Minimize & $0.6 \pm 0.983 \%$ & $3.5 \pm 2.8$ & $<0.01$ \\
\hline $\mathrm{D}_{1 \%}[\mathrm{~Gy}]$ & Minimize & $6.3 \pm 6.6$ & $11.9 \pm 5.3$ & $<0.01$ \\
\hline \multicolumn{5}{|c|}{ Right breast: $494.6 \pm 247.4 \mathrm{~cm}^{3}$} \\
\hline Mean [Gy] & Minimize & $0.7 \pm 1.781 \%$ & $3.7 \pm 3.8$ & $<0.01$ \\
\hline$D_{1 \%}[G y]$ & Minimize & $4.9 \pm 9.2$ & $1.9 \pm 7.3$ & $<0.01$ \\
\hline \multicolumn{5}{|c|}{ Lungs: $4540.0 \pm 1538.3 \mathrm{~cm}^{3}$} \\
\hline Mean [Gy] & $<15 G y$ & $4.3 \pm 1.846 \%$ & $7.9 \pm 3$ & $<0.01$ \\
\hline $\mathrm{D}_{1 \%}[\mathrm{~Gy}]$ & Minimize & $30.0 \pm 0.6$ & $28.7 \pm 1.0$ & $<0.01$ \\
\hline$V_{20 G y}[\%]$ & $<20 \%$ & $6.7 \pm 3.4$ & $9.8 \pm 7.5$ & $<0.01$ \\
\hline$V_{15 G y}[\%]$ & Minimize & $9.7 \pm 5.0$ & $18.2 \pm 13.1$ & $<0.01$ \\
\hline$V_{10 G y}[\%]$ & Minimize & $15.0 \pm 7.5$ & $32.9 \pm 18.6$ & $<0.01$ \\
\hline$V_{5 G y}[\%]$ & Minimize & $27.8 \pm 11.7$ & $52.7 \pm 18.8$ & $<0.01$ \\
\hline \multicolumn{5}{|c|}{ Heart $554.8 \pm 146.2 \mathrm{~cm}^{3}$} \\
\hline Mean [Gy] & Minimize & $4.1 \pm 3.938 \%$ & $6.6 \pm 4.6$ & $<0.01$ \\
\hline $\mathrm{D}_{1 \%}[\mathrm{~Gy}]$ & Minimize & $26.3 \pm 8.9$ & $26.8 \pm 8.6$ & $<0.01$ \\
\hline \multicolumn{5}{|c|}{ Heart wall $133.6 \pm 25.9 \mathrm{~cm}^{3}$} \\
\hline Mean [Gy] & $<5 G y$ & $2.9 \pm 4.040 \%$ & $4.9 \pm 4.7$ & $<0.01$ \\
\hline $\mathrm{D}_{1 \%}[\mathrm{~Gy}]$ & Minimize & $20.8 \pm 11.5$ & $21.9 \pm 10.9$ & 0.05 \\
\hline \multicolumn{5}{|l|}{ Healthy tissue } \\
\hline Mean [Gy] & Minimize & $2.3 \pm 1.549 \%$ & $4.5 \pm 2.1$ & $<0.01$ \\
\hline$V_{10 G y}[\%]$ & Minimize & $8.5 \pm 6.848 \%$ & $16.3 \pm 10.7$ & $<0.01$ \\
\hline $\mathrm{Cl}_{90 \%}$ & Minimize & $1.2 \pm 0.1$ & $1.2 \pm 0.1$ & n.s. \\
\hline
\end{tabular}

$D_{\mathrm{x} \%}$ dose received by the $\mathrm{x} \%$ of the volume, $V_{\mathrm{x} \%}$ volume receiving at least $\mathrm{x} \%$ of the prescribed dose, $\mathrm{Cl}$ ratio between the patient volume receiving at least $90 \%$ of the prescribed dose and the volume of the total PTV

The DVH were assessed quantitatively using a number of appropriate metrics, which included the mean dose $\left(D_{\text {mean }}\right)$, the dose received by $1 \%$ of the PTV/OAR's volume $\left(D_{1 \%}\right), D_{98 \%}, D_{95 \%}, D_{5-95 \%}$, as well as a variety of $\mathrm{V}_{\mathrm{xGy}}$ values. For each parameter analyzed, the mean values \pm SD over the 2 patient cohorts were analyzed. In addition, the conformity index $\left(\mathrm{CI}_{90 \%}\right)$, which measures the degree of radiation conformity and should be ideally equal to 1 , if the PTV matches exactly the $90 \%$ isodose volume, was assessed.

The Wilcoxon matched-paired signed-rank test was applied to evaluate the level of significance of the observed differences between the dose-volume metrics. The threshold for statistical significance was set at $<0.05$. The statistical analysis was performed on the Statistical Package for Social Sciences system (SPSS, Ver.22.0, IBM Corp). 
Proton dose perturbation (PDP) and statistical analyses PDP analysis was performed to investigate one of the possible dosimetric concerns that could be simulated by the TPS. PDP could in fact result from CT calibration errors of the therapeutic protons. The calibration errors were simulated by varying the Hounsfield Units (HU) to stopping power calibration by applying a \pm 3 and $\pm 4 \%$ error in CT numbers [31]. His primarily mimics the proton penetration into the $\mathrm{HL}$ patient. The analysis was conducted on all OARs and target volumes to appraise the possible loss in coverage or increase in involvement due to these uncertainties.

\section{Results}

\section{Dose comparative study}

Both IMPT and VMAT provided excellent coverage of the planning tumor volume (PTV). No difference was observed with regard to the mean dose to the PTV. Although both techniques fulfilled the planning constraints for CTV and PTV, IMPT provided better conformity, homogeneity and a slightly better coverage. Typical dose distributions in axial, coronal and sagittal planes for one patient and the two techniques are illustrated in Fig. 1. The color-wash display was set to 5-40 Gy with the significantly reduced volume of the low dose bath with IMPT.

The numerical analysis conducted on the dose-volume histograms (DVH) for the various parameters considered are summarized in Table 2 for the targets and in Table 3 for the OARs. The planning strategy adopted for both proton and photon plans, allowed to achieve the level of target coverage required by the study aims. More than $95 \%$ of the prescribed dose was in fact computed for the $98 \%$ of CTV and more than $90 \%$ of the dose was estimated to more than $95 \%$ of the PTV. The target homogeneity was expressed by means of $D_{5 \%}-D_{95 \%}$ and this resulted of the order of 2 Gy for IMPT plans and 2.5 Gy for the photons (relatively to the CTV); both findings demonstrate a very high homogeneity of the dose distributions. Although most of the parameters investigated showed statistically significant differences between IMPT and VMAT, the absolute relevance of these might be clinically minor.

Concerning OAR sparing, the data revealed that IMPT led to a statistically significant reduction of mean doses by $38-83 \%$ to the lung, the breasts (female patients), the heart and the heart wall (Table 3). IMPT led also to a statistically significant reduction in the near-to-maximum dose in the spinal cord (about 4 Gy of further sparing with IMPT compared to VMAT). Concerning the lungs, all the sub-volumes covered with 5, 10, 15, 20, and 30 Gy could be significantly reduced with IMPT (Table 3 and Fig. 2) while the mean lung dose was nearly halved. Also, the mean doses to the heart and heart wall could be reduced by more than $30 \%$. In particular the average of the mean dose to the entire heart could be reduced to 4 Gy (compared to almost $7 \mathrm{~Gy}$ for the photon case). The mean dose and low dose bath $\left(\mathrm{V}_{10 \mathrm{~Gy}}\right)$ to the healthy tissue could be reduced by nearly $50 \%$ (Table 3 ).

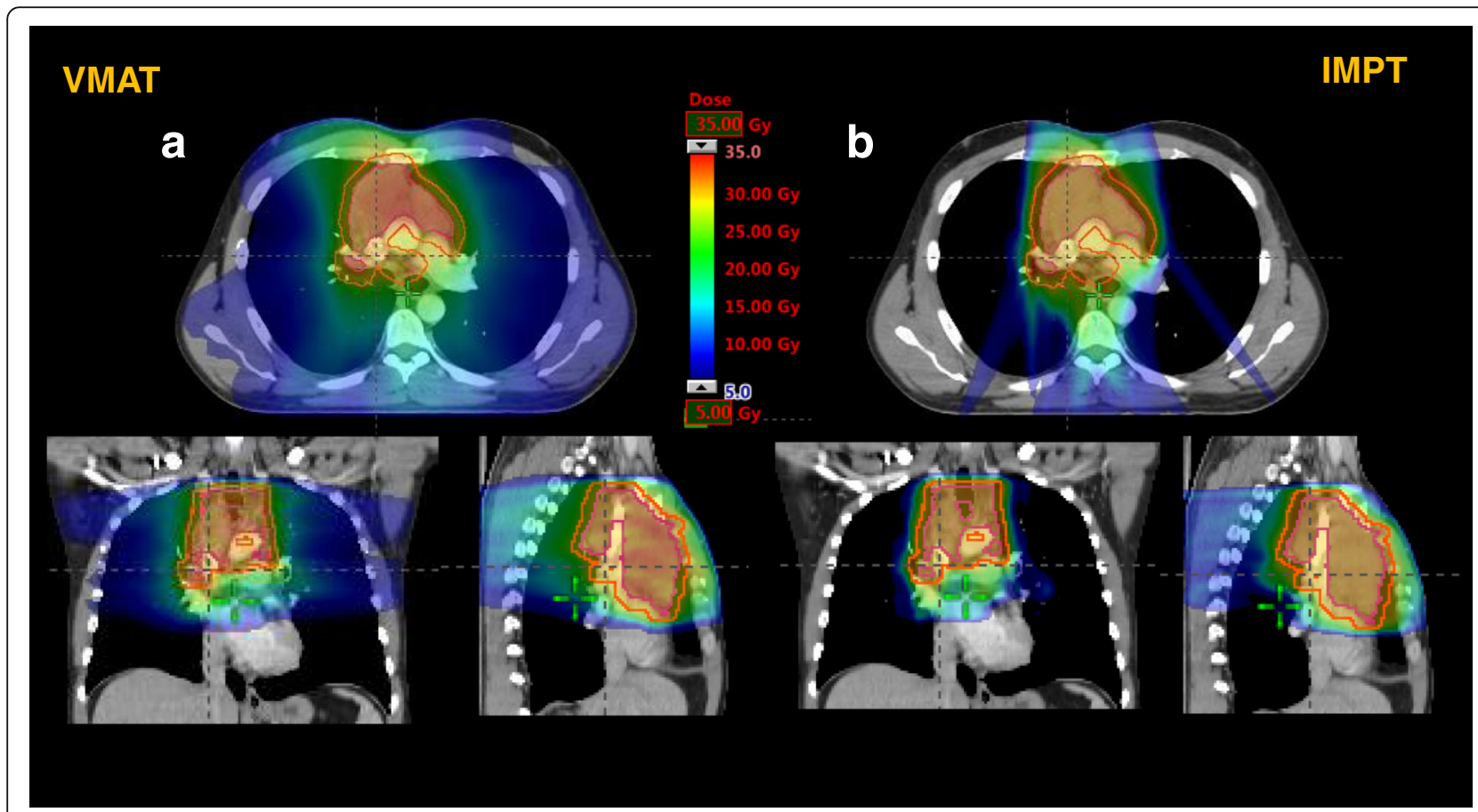

Fig. 1 a Volumetric arc treatment of a patient with large mediastinal mass with a considerable low does bath (blue). b Intensity modulated proton therapy with a significant reduction of low dose bath 

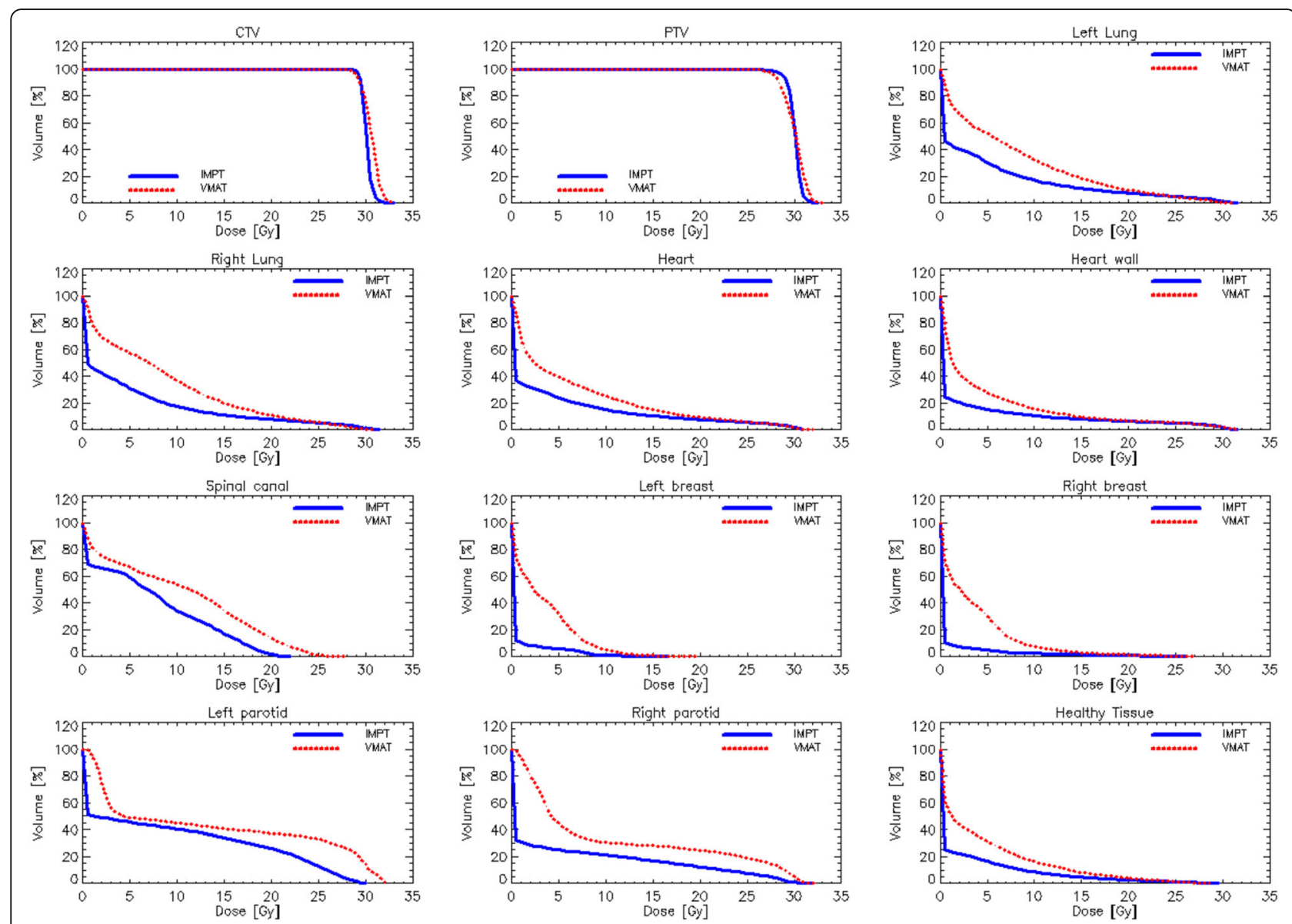

Fig. 2 Comparison of dose volume histograms for VMAT (photons, Blue) and IMPT (Red) for CTV, PTV, heart wall, lungs, spinal cord, breast, parotid gland, healthy tissue

\section{Proton dose perturbation (PDP)}

Despite the simulated 3 and $4 \%$ miscalibration errors, no remarkable or measurable impact was observed on the organs at risk. In contrast, the effect of range uncertainties was quite relevant for the PTV, although almost completely mitigated for CTV volumes (Table 4). A magnified display of the average DVH for the CTV and PTV and the various CT calibration errors simulated is shown in Fig. 3.

\section{Discussion}

This study is one of the first to evaluate intensity-modulated photon (IMRT/VMAT) and intensity modulated proton radiation (IMPT) techniques in deep inspiration breath hold $(\mathrm{DIBH})$ in $\mathrm{HL}$ patients with mediastinal involvement. Depending on the stage, we applied the involved field concepts analogous to the HD16 and HD17 study, or the concept of irradiating PET positive remaining lymphoma tissue analogous to the HD18 study [32], including only sites originally involved in the treatment volume. Despite the simulated 3 and $4 \%$ miscalibration errors, no remarkable or measurable impact was observed on the organs at risk. We could demonstrate the superiority of IMPT over highly sophisticated photon treatment: Reductions in the mean dose, and absolute or relative volumes of the non-targeted tissues were observed for healthy tissue, lung, and breast tissue. The

Table 4 Summary of target volumes dosimetric analysis from the PDP analysis. Data are reported only for the positive miscalibrations since these represent the worst scenarios

\begin{tabular}{llll}
\hline Parameter & reference & $\Delta(+3 \%)$ & $\Delta(+4 \%)$ \\
\hline CTV & & & \\
Mean [Gy] & $30.1 \pm 0.2$ & $29.9 \pm 0.2-0.7 \%$ & $29.9 \pm 0.3-0.7 \%$ \\
D $_{95 \%}[G y]$ & $29.4 \pm 0.1$ & $28.9 \pm 0.3-1.7 \%$ & $28.5 \pm 0.4-3.1 \%$ \\
D98\% [Gy] & $29.4 \pm 0.1$ & $28.4 \pm 0.4-3.4 \%$ & $27.8 \pm 0.6-5.4 \%$ \\
PTV & & \\
Mean [Gy] & 30.0 & $29.7 \pm 0.2-1.0 \%$ & $29.4 \pm 0.3-2.0 \%$ \\
D $_{95 \%}[G y]$ & $28.8 \pm 0.3$ & $27.6 \pm 0.5-4.2 \%$ & $26.7 \pm 0.7-7.3 \%$ \\
D $_{98 \%}[G y]$ & $28.8 \pm 0.3$ & $26.4 \pm 0.8-8.3 . \%$ & $25.3 \pm 1.0-12.2 \%$ \\
\hline$D_{x \%}$ dose received by the $\times \%$ of
\end{tabular}

$D_{\mathrm{x} \%}$ dose received by the $\mathrm{x} \%$ of the volume, $V_{\mathrm{x} \%}$ volume receiving at least $\mathrm{x} \%$ of the prescribed dose, $\mathrm{Cl}$ ratio between the patient volume receiving at least $90 \%$ of the prescribed dose and the volume of the total PTV 

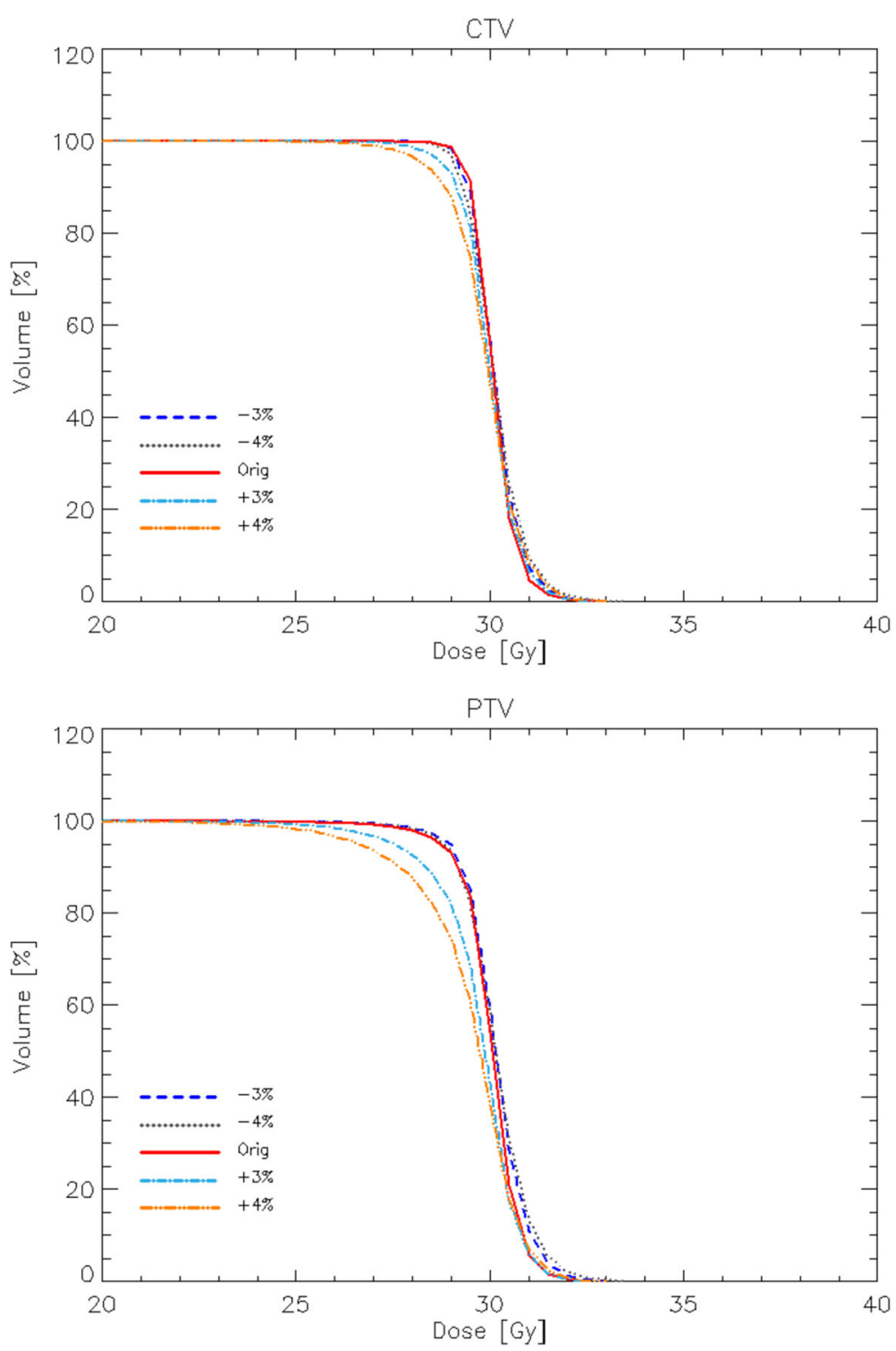

Fig. 3 Average DVH for the simulated CTV, PTV and various CT calibration errors

relative reduction (DIBH-VMAT versus IMPT) in mean body, lung, and breast doses was 38-83\%.

A critical issue for all planning comparisons is identifying the organs at risk and prioritizing the sparing of those structures. Our results confirm prior retrospective data, which indicated substantial benefit to selected organs when conventional proton radiation was compared with either conventional 3-D-CRT or conventional IMRT in patients with $\mathrm{HL}$, depending on optimization criteria [18, 19, 21, 24, 26, 28]. Importantly, we could show improvements due to the mean dose to the heart, lung, and breasts in all patients when comparing IMPT against the most comprehensive and sophisticated photon techniques in deep inspiration breath hold. The gantry angles chosen for the plans might play a role as well and, although a class solution was applied, some individualization was applied in the tuning of the angles to minimize the OARs involvement. In some cases, this might have been further improved with completely different approaches but would have also introduced further heterogeneity in the data.

As an example of the adequate planning strategy adopted for IMPT, our findings, both in term of target coverage and organs at risk sparing are consistent and equivalent within the uncertainties with other data from literature, in particular the study from Zeng et al. [33].

Several publications have evaluated the impact of DIBH techniques. Inflating the lungs and pulling the heart downward could reduce doses to the heart and the lungs. Therefore, DIBH can be considered as a standard 
technique for radiation in patients with mediastinal involvement in HL $[10,23,34]$. The impact of patients' compliance in the DIBH process might be particularly severe for proton plans. The dosimetric relevance of a sub-optimal DIBH condition was not investigated in this study, due to technical limitations of the tools available and the impossibility to simulate multiple CT scans with different levels of breath hold. It is here acknowledged that, proton delivery systems should be equipped with appropriated breath monitoring system and that the compliance from patients might play a relevant role. To mention also the fact that not all currently existing proton facilities are equipped with volumetric imaging capabilities while the ProScan system, applied for our study, has this functionality.

The benefit from proton techniques depends on the individual anatomy and location of the HL. Here, we selected patients with mediastinal HL involvement. Although patients with involvement in the superior mediastinum benefit less from proton therapy because the volume of heart irradiated is already minimal [19], even in these situations our results show the benefit of IMPT in sparing mean heart and breast doses.

Different cardiac (sub-) structures are used for dose-volume histogram analysis in planning $[19,26]$. The origin of the coronary arteries or distinct single vessels is related to many uncertainties with regard to definition and contouring on planning $\mathrm{CT}$ and organ movement during treatment. The importance of contouring and localization and its clinical importance has to be questioned and remains to be been validated. Furthermore, the clinical implication of dose reduction to the different cardiac subunits is still unclear.

Nevertheless, the dose to the heart is a great concern in HL patients. Cardiac magnetic resonance imaging (MRI) performed on long-term survivors after radiation demonstrated changes in valvula function or ventricular function in up to $68 \%$ of the patients [5]. Tukenova et al. [35] and Mulrooney et al. [36] showed that the risk of death from cardiac disease was significantly higher in patients who received cardiac doses of 5 to 15 Gy. Data from the Childhood Cancer Survivor Study cohort confirmed a correlation between dose and cardiac late effects. The excess relative risk of developing cardiac disease per Gy was 0.49 (95\% CI, 0.26-1.3) in patients who had not received anthracycline-containing chemotherapy. Also, smoking status and Body Mass Index influence this risk [37]. For plan optimization we tried to minimize the mean dose to the heart (wall). In our study, the proton technique leads to a statistically significant reduction in the mean doses.

According to the available data [35-37] our planning goal to decrease the mean dose to the heart to $<5 \mathrm{~Gy}$, could only be reached with IMPT, and not with VMAT.
Whether the reduction we achieved, i.e. from $4.9 \pm 4.7$ Gy with photons down to $2.9 \pm 4.0$ Gy with protons, is of clinical relevance remains unclear. Thus, close follow-up of these patients treated with protons will be important. However, we expect a better long-term result with regard to cardiac complications, although no data is as yet available for risk reduction associated with mean heart doses < 5 Gy.

Radiation-induced secondary malignancies appear to be a major cause of mortality in HL populations. The majority of secondary malignancies have been observed at the radiation field border [38, 39]. Due to the low dose bath, IMRT is likely to double the incidence of secondary malignancies compared to conventional radiotherapy, from about 1 to $1.75 \%$ for patients surviving 10 years [40]. As the radiation dose increases, radiation-induced cell death may become dominant over carcinogenic mutations. Protons with their sharp dose gradient should theoretically be able to reduce the risk of secondary cancer. Discussions are ongoing about the shape of the dose-response relationship for higher doses and the risk of secondary malignancies [17]. In a recently published case report Meyer and colleagues showed that proton beam therapy allowed a better target coverage, a better dose homogeneity and conformity to the planning target volume. Furthermore it reduced volume of healthy tissues receiving low doses although it increases weakly volume of tissues receiving high doses [41].

Travis et al. [40] have shown an increased risk of secondary lung cancer after doses as low as 5 Gy. Excess risk after radiotherapy began 5 yrs after treatment and persisted for more than 20 years. For the lungs, our IMPT procedure led to a $50 \%$ reduction in nearly all analyzed sub-volumes (V5, V10, V15, V20) compared with photon treatment. With a mean dose reduction to $4.3 \pm 1.8$ Gy with protons we may have reached uncritical dose levels with regard to the available data.

A large proportion of the second malignancies observed in HL survivors are breast cancers, which occur primarily in women treated for $\mathrm{HL}<30$ years of age, suggesting a need to prioritize breast dose in these patients [19]. The risk for breast cancer depends on the age at radiation exposition, the volume, and the dose. Childhood cancer survivors treated with lower delivered doses of radiation to a large volume had a high risk of breast cancer compared to patients treated with high doses to smaller volumes [7]. Travis et al. [40] have observed a 3.2-fold increase in breast cancers with a radiation dose of $\geq 4$ Gy compared to HL patients who received lower doses. Interestingly, even DIBH techniques with photons provided a mean dose $<4$ Gy in our patients, specifically doses of $3.5 \pm 2.8$ or $3.7 \pm 3.8$ Gy were recorded to the left or right breast with VMAT. In contrast to Hoppe 
et al. [19] with conventional proton planning, in our study IMPT substantially decreased the mean dose even further to $0.6 \pm 0.9$ Gy to the left and $0.7 \pm 1.7$ to the right breast $(p<0.001)$.

Naturally uncertainties still remain as regard to secondary cancer after radiation, which will require long-term studies. Moreover, looking at certain DVH parameters does not take into account different factors, e.g. relative biological effectiveness of the radiation, the radiation type (e.g. photons versus protons), details of the beam delivery (e.g. scanned versus scattered proton beams), and the age, gender and other treatments (chemotherapy) of the patient [19].

As a final point of discussion, we would clarify that we opted for a simple comparison between IMPT and VMAT and did not included in the study fixed field IMRT. Indeed, the comparison between VMAT and IMRT was already performed and reported in [17, 35]. In those studies, we did not find any relevant or clinically significant difference which might support the use of IMRT over VMAT. In particular for breast and lungs, the data showed that VMAT could slightly reduce their involvement as compared to IMRT. Concerning the risk of secondary cancer induction, with both linear and not linear models we showed that ERR for VMAT is slightly lower than for IMRT in both involved nodes or involved field settings.

\section{Conclusion}

Even compared with highly sophisticated DIBH VMAT techniques in a very experienced treatment center, IMPT provided superior coverage while reducing the mean doses to the breasts, lungs, heart and cardiac wall. Long-term follow-up is needed to confirm the benefits of IMPT over X-ray techniques in terms of late toxicity and secondary malignancies. Regardless of the planning paradigm used, range uncertainties can substantially under dose the PTV, although this might not lead to clinically significant deterioration of CTV coverage. With the geometry applied here, no impact was observed on organs at risk, indicating that IMPT could play an important role in reducing the risk of inducing secondary malignancies such as breast cancer.

\footnotetext{
Abbreviations

3D-PRT 3D: Proton treatment; Cl: Conformity index; CT: Computer tomography; CTV: Clinical target volume; DIBH: Deep inspiration breathhold DVH: Dose volume histogram; EORTC: European Organization for Research and Treatment of Cancer; GHSG: German Hodgkin Study Group; GTV: Gross tumor volume; Gy: Gray; HL: Hodgkin lymphoma; HT: Helical tomotherapy; HU: Hounsfield units; IFRT: Involved field radiotherapy; IMPT: Intensity modulated Proton Therapy; IMRT: Intensity modulated Radiotherapy; INRT: Involved node radiotherapy; MeV: Megaelectron volt; OAR: Organs at risk; PDP: Perturbation proton dose; PRT: Proton treatment; PTV: Planning tumor volume; SPSS: Statistic package for social science system; VMAT: Volumetric modulated Arc Therapy; VMS: Varian medical systems
}

Availability of data and materials

Please contact author for data requests.

\section{Authors' contributions}

CB delineated all target lesions and OAR's in planning CT scans. This was done in professional exchange with SM and KJ.WB and AV were responsible for IMRT calculating and LC, AF and MS performed the IMPT calculation. The statistics of this analysis were performed by LC. SM and CB were responsible for writing the manuscript in critical discussion with LC, AE and WB. All the authors read and approved the manuscript.

Ethics approval and consent to participate

Not applicable.

\section{Consent for publication \\ Not applicable.}

\section{Competing interests}

Dr. L. Cozzi acts as Scientific Advisor to Varian Medical Systems. Other authors have no conflict of interest.

\section{Publisher's Note}

Springer Nature remains neutral with regard to jurisdictional claims in published maps and institutional affiliations.

\section{Author details \\ ${ }^{1}$ Medical Faculty, Department of Radiation Oncology, CyberKnife Center and Radiation Reference Center of the GHSG, University of Cologne, Kerpener Str. 52, 50937 Köln, Cologne, Germany. ${ }^{2}$ Medical Faculty, Department of Hematology and Oncology, University of Cologne, Cologne, Germany. ${ }^{3}$ German Hodgkin Study Group, Cologne, Germany. ${ }^{4}$ Radiotherapy and Radiosurgery, Humanitas Cancer Center and Research Hospital, Milan, Italy. ${ }^{5}$ Department of Biomedical Sciences, Humanitas University, Milan, Italy.}

Received: 2 February 2018 Accepted: 15 June 2018 Published online: 03 July 2018

References

1. Behringer $K$, Josting A, Schiller P, Eich HT, Bredenfeld H, Diehl V, et al. Solid tumors in patients treated for Hodgkin's disease: a report from the German Hodgkin Lymphoma Study Group. Ann Oncol. 2004;15:1079-85. Available from: https://academic.oup.com/annonc/article-lookup/doi/10.1093/annonc/ mdh273.

2. Ng AK, Bernardo MP, Weller E, Backstrand KH, Silver B, Marcus KC, et al. Long-term survival and competing causes of death in patients with earlystage Hodgkin's disease treated at age 50 or younger. J Clin Oncol. 2002;20: 2101-8. Available from: http://ascopubs.org/doi/10.1200/JCO.2002.08.021.

3. Aleman BMP, van den Belt-Dusebout AW, Klokman WJ, Van't Veer MB, Bartelink H, van Leeuwen FE. Long-Term Cause-Specific Mortality of Patients Treated for Hodgkin's Disease. J Clin Oncol. 2003;21:3431-9. Available from: http://ascopubs.org/doi/10.1200/jco.2003.07.131.

4. Maraldo MV, Brodin NP, Aznar MC, Vogelius IR, Munck af Rosenschöld P, Petersen PM, et al. Estimated risk of cardiovascular disease and secondary cancers with modern highly conformal radiotherapy for early-stage mediastinal Hodgkin lymphoma. Ann Oncol. 2013;24:2113-8. Available from: https://academic.oup.com/annonc/article-lookup/doi/10.1093/annonc/ mdt156.

5. Machann W, Beer M, Breunig M, Störk S, Angermann C, Seufert I, et al. Cardiac magnetic resonance imaging findings in 20-year survivors of mediastinal radiotherapy for Hodgkin's disease. Int J Radiat Oncol Biol Phys. 2011;79:1117-23. Available from: http://linkinghub.elsevier.com/retrieve/pii/ S0360301610000039.

6. Küpeli S, Hazırolan T, Hazirolan T, Varan A, Akata D, Alehan D, et al. Evaluation of coronary artery disease by computed tomography angiography in patients treated for childhood Hodgkin's lymphoma. J Clin Oncol. 2010;28:1025-30. Available from: http://ascopubs.org/doi/10.1200/ JCO.2009.25.2627.

7. Moskowitz CS, Chou JF, Wolden SL, Bernstein JL, Malhotra J, Novetsky Friedman D, et al. Breast Cancer After Chest Radiation Therapy for Childhood Cancer. J Clin Oncol. 2014;32:2217-23. Available from: http:// ascopubs.org/doi/10.1200/JCO.2013.54.4601. 
8. Pinnix CC, Abou Yehia Z, Smith GL, Milgrom SA, Ho JC, Reddy J, et al. More chemotherapy does not obviate the need for radiation therapy (RT): treatment for early-stage favorable Hodgkin lymphoma according to the HD10 trial compared with chemotherapy alone. Int J Radiat Oncol Biol Phys. 2016;96:E489-90. Available from: http://linkinghub.elsevier.com/retrieve/pii/ S0360301616321848.

9. Radford J, Illidge T, Counsell N, Hancock B, Pettengell R, Johnson P, et al. Results of a Trial of PET-Directed Therapy for Early-Stage Hodgkin's Lymphoma. N Engl J Med. 2015;372:1598-607. Available from: http://www. nejm.org/doi/10.1056/NEJMoa1408648.

10. Voong K, McSpadden K, Pinnix CC, Shihadeh F, Reed V, Salehpour MR, et al. Dosimetric advantages of a "butterfly" technique for intensity-modulated radiation therapy for young female patients with mediastinal Hodgkin's lymphoma. Radiat Oncol. 2014;9:94-9.

11. Specht L, Yahalom J, Illidge T, et al. Modern radiation therapy for Hodgkin lymphoma: field and dose guidelines from the international lymphoma radiation oncology group (ILROG). Int J Radiat Oncol Biol Phys. 2014;89:85462. Available from: https://doi.org/10.1016/j.jirobp.2013.05.005.

12. Oguchi M, Hasegawa M, Ishibashi N, Isobe K, Imai M, Ejima Y, et al. ILROG guideline of radiation therapy for nodal lymphomas: involved site radiation therapy. [Rinsho ketsueki] Jpn J Clin Hematol. 2014;55:1903-11. Available from: http://eutils.ncbi.nlm.nih.gov/entrez/eutils/elink.fcgi?dbfrom= pubmed\&id $=25297754 \&$ retmode $=$ ref\&cmd $=$ prlinks.

13. Engert A, Plütschow A, Eich HT, Lohri A, Dörken B, Borchmann $P$, et al. Reduced treatment intensity in patients with early-stage Hodgkin's lymphoma. N Engl J med. 2010;363:640-52. Available from: http://www. nejm.org/doi/abs/10.1056/NEJMoa1000067.

14. Lohr F, Georg D, Cozzi L, Eich HT, Weber DC, Koeck J, et al. Neue Strahlentherapietechniken für Involved-Field- und Involved-Node-Behandlung des mediastinalen Hodgkin-Lymphoms. Strahlenther Onkol. 2014;190:864-71. Available from: http://link.springer.com/10.1007/s00066-014-0719-9.

15. Engert A, Schiller $P$, Josting A, Herrmann R, Koch P, Sieber M, et al. Involvedfield radiotherapy is equally effective and less toxic compared with extended-field radiotherapy after four cycles of chemotherapy in patients with early-stage unfavorable Hodgkin's lymphoma: results of the HD8 trial of the German Hodgkin's lymphoma study group. J Clin Oncol. 2003;21: 3601-8. Available from: http://ascopubs.org/doi/10.1200/JCO.2003.03.023.

16. Verhappen MH, Poortmans PMP, Raaijmakers E, Raemaekers JMM. Reduction of the treated volume to involved node radiation therapy as part of combined modality treatment for early stage aggressive non-Hodgkin's lymphoma. Radiother Oncol. 2013;109:133-9. Available from: http:// linkinghub.elsevier.com/retrieve/pii/S0167814013003885.

17. Weber DC, Johanson S, Peguret N, Cozzi L, Olsen DR. Predicted risk of radiation-induced cancers after involved field and involved node radiotherapy with or without intensity modulation for early-stage Hodgkin lymphoma in female patients. Int J Radiat Oncol Biol Phys. 2011;81:490-7. Available from: http://linkinghub.elsevier.com/retrieve/pii/S0360301610007583.

18. Hoppe BS, Flampouri S, Su Z, Morris CG, Latif N, Dang NH, et al. Consolidative involved-node proton therapy for stage IA-IIIB mediastinal Hodgkin lymphoma: preliminary Dosimetric outcomes from a phase ॥ study. Int J Radiat Oncol Biol Phys. 2012;83:260-7. Available from: http:// linkinghub.elsevier.com/retrieve/pii/S0360301611028252.

19. Hoppe BS, Flampouri S, Su Z, Latif N, Dang NH, Lynch J, et al. Effective dose reduction to cardiac structures using protons compared with 3DCRT and IMRT in mediastinal Hodgkin lymphoma. Int J Radiat Oncol Biol Phys. 2012; 84:449-55. Available from: http://linkinghub.elsevier.com/retrieve/pii/ S0360301611037217.

20. Filippi AR, Ragona R, Piva C, Scafa D, Fiandra C, Fusella M, et al. Optimized volumetric modulated arc therapy versus 3D-CRT for early stage mediastinal Hodgkin lymphoma without axillary involvement: a comparison of second cancers and heart disease risk. Int J Radiat Oncol Biol Phys. 2015;92:161-8. Available from: http://linkinghub.elsevier.com/ retrieve/pii/S0360301615002187

21. Chera BS, Rodriguez C, Morris CG, Louis D, Yeung D, Li Z, et al. Dosimetric comparison of three different involved nodal irradiation techniques for stage II Hodgkin's lymphoma patients: conventional radiotherapy, intensitymodulated radiotherapy, and three-dimensional proton radiotherapy. Int J Radiat Oncol Biol Phys. 2009:75:1173-80. Available from: https://doi.org/10. 1016/j.jijobp.2008.12.048.

22. Besson N, Pernin V, Zefkili S, Kirova YM. Evolution of radiation techniques in the treatment of mediastinal lymphoma: from 3D conformal radiotherapy
(3DCRT) to intensity-modulated RT (IMRT) using helical tomotherapy (HT): a single-centre experience and review of the literature. Br J Radiol. 2016;89: 20150409-7. Available from: http://www.birpublications.org/doi/10.1259/bjr. 20150409.

23. Aznar MC, Maraldo MV, Schut DA, Lundemann M, Brodin NP, Vogelius IR, et al. Minimizing late effects for patients with mediastinal Hodgkin lymphoma: deep inspiration breath-hold, IMRT, or both? Int J Radiat Oncol Biol Phys. 2015;92:169-74. Available from: https://doi.org/10.1016/j.jijobp. 2015.01.013.

24. Hoppe BS, Flampouri S, Zaiden R, Slayton W, Sandler E, Ozdemir S, et al. Involved-node proton therapy in combined modality therapy for Hodgkin lymphoma: results of a phase 2 study. Int J Radiat Oncol Biol Phys. 2014;89: 1053-9. Available from: http://linkinghub.elsevier.com/retrieve/pii/ S0360301614005008

25. Hoppe BS, Hill-Kayser CE, Tseng YD, Flampouri S, Elmongy HM, Cahlon O, et al. Consolidative proton therapy after chemotherapy for patients with Hodgkin lymphoma. Ann Oncol. 2017;28:2179-84. Available from: http:// academic.oup.com/annonc/article/doi/10.1093/annonc/mdx287/4082165/ Consolidative-proton-therapy-after-chemotherapy

26. Li J, Dabaja B, Reed V, Allen PK, Cai H, Amin MV, et al. Rationale for and preliminary results of proton beam therapy for mediastinal lymphoma. Int J Radiat Oncol Biol Phys. 2011;81:167-74. Available from: http://linkinghub. elsevier.com/retrieve/pii/S0360301610006656

27. Zhang R, Howell RM, Homann K, Giebeler A, Taddei PJ, Mahajan A, et al. Predicted risks of radiogenic cardiac toxicity in two pediatric patients undergoing photon or proton radiotherapy. Radiat Oncol. 2013;8:184. Available from: http://ro-journal.biomedcentral.com/articles/10.1186/1748717X-8-184

28. Horn S, Fournier-Bidoz N, Pernin V, Peurien D, Vaillant M, Dendale R, et al. Comparison of passive-beam proton therapy, helical tomotherapy and 3D conformal radiation therapy in Hodgkin's lymphoma female patients receiving involved-field or involved site radiation therapy. Cancer Radiother. 2016;20:98-103. Available from: http://linkinghub.elsevier.com/retrieve/pii/ S127832181600038X

29. Rechner LA, Maraldo MV, Vogelius IR, Zhu XR, Dabaja BS, Brodin NP, et al. Life years lost attributable to late effects after radiotherapy for early stage Hodgkin lymphoma: the impact of proton therapy and/or deep inspiration breath hold. Radiother Oncol. 2017;125:41-7.

30. Hoppe BS, Mendenhall NP, Louis D, Li Z, Flampouri S. Comparing breath hold and free breathing during intensity-modulated radiation therapy and proton therapy in patients with mediastinal Hodgkin lymphoma. International Journal of Particle Therapy. 2017:3:492-6.

31. Lomax AJ. Intensity modulated proton therapy and its sensitivity to treatment uncertainties 2: the potential effects of inter-fraction and inter-field motions. Phys Med Biol. 2008;53:1043-56. Available from: http://stacks.iop.org/0031-9155/53/i=4/a=015?key=crossref. 49a5dabe348f26645479f8d68643b59d

32. Eich HT, Müller R-P, Engenhart-Cabillic R, Lukas $P$, Schmidberger $H$, et al. Involved-Node-Radiotherapie in frühen Stadien des Hodgkin-Lymphoms. Definition und Richtlinien der Deutschen Hodgkin-LymphomStudiengruppe (GHSG). Strahlenther Onkol. 2008;184:406-10. Available from: http://link.springer.com/10.1007/s00066-008-1882-7

33. Zeng C, Plastaras JP, James P, Tochner ZA, Hill-Kayser CE, Hahn SM, et al. Proton pencil beam scanning for mediastinal lymphoma: treatment planning and robustness assessment. Acta Oncologica. 2016;55:1132-8.

34. Paumier A, APM D, MGM D, Ghalibafian M, Gilmore J, JGM D, et al. Dosimetric benefits of intensity-modulated radiotherapy combined with the deep-inspiration breath-hold technique in patients with mediastinal Hodgkin's lymphoma. Int J Radiat Oncol Biol Phys. 2012;82:1522-7. Available from: https://doi.org/10.1016/j.jijrobp.2011.05.015.

35. Tukenova M, Guibout C, Oberlin O, Doyon F, Mousannif A, Haddy N, et al. Role of Cancer Treatment in Long-Term Overall and Cardiovascular Mortality After Childhood Cancer. J Clin Oncol. 2010;28:1308-15. Available from: http://ascopubs.org/doi/10.1200/JCO.2008.20.2267.

36. Mulrooney DA, Mulrooney DA, Yeazel MW, Yeazel MW, Kawashima T, Kawashima T, et al. Cardiac outcomes in a cohort of adult survivors of childhood and adolescent cancer: retrospective analysis of the childhood Cancer survivor study cohort. BMJ. 2009;339:b4606. Available from: http:// www.bmj.com/cgi/doi/10.1136/bmj.b4606.

37. Haddy N, Diallo S, El-Fayech C, Schwartz B, Pein F, Hawkins M, et al. Cardiac Diseases Following Childhood Cancer Treatment CLINICAL PERSPECTIVE. 
Circulation. 2015;133:31-8. Available from: http://circ.ahajournals.org/lookup/ doi/10.1161/CIRCULATIONAHA.115.016686.

38. Epstein R, Hanham I, Dale R. Radiotherapy-induced second cancers: are we doing enough to protect young patients? Eur J Cancer (1990). 1997;33:52630. Available from: http://eutils.ncbi.nlm.nih.gov/entrez/eutils/elink. fcgi?dbfrom=pubmed\&id=9274430\& retmode=ref\&cmd=prlinks

39. Dörr W, Herrmann T. Second primary tumors after radiotherapy for malignancies. Treatment-related parameters. Strahlenther Onkol. 2002;178 357-62. Available from: http://eutils.ncbi.nlm.nih.gov/entrez/eutils/elink. fcgi?dbfrom=pubmed\&id=12163989\&retmode=ref\&cmd=prlinks.

40. Travis LB, Gospodarowicz M, Curtis RE, Clarke EA, Andersson M, Glimelius B et al. Lung cancer following chemotherapy and radiotherapy for Hodgkin's disease. J Natl Cancer Inst. 2002;94:182-92. Available from: http://eutils.ncbi. nlm.nih.gov/entrez/eutils/elink.fcgi?dbfrom=pubmed\&id= 11830608\&retmode=ref\&cmd=prlinks.

41. Meyer E, Goudjil F, Tamburini J, Dendale R, Kirova Y. Proton beam therapy for the management of mediastinal Hodgkin lymphoma in a young female patient. Cancer Radiother. 2018;

Ready to submit your research? Choose BMC and benefit from:

- fast, convenient online submission

- thorough peer review by experienced researchers in your field

- rapid publication on acceptance

- support for research data, including large and complex data types

- gold Open Access which fosters wider collaboration and increased citations

- maximum visibility for your research: over $100 \mathrm{M}$ website views per year

At BMC, research is always in progress.

Learn more biomedcentral.com/submissions 